\title{
A Novel Control of a Reverse Osmosis Desalination System Powered by Photovoltaic Generator
}

\author{
Abderrahmen BEN CHAABENE \\ Research Unit C3S \\ School of Sciences and Technologies, ESSTT \\ Tunis, Tunisia \\ abd.chaabene@yahoo.fr
}

\author{
Anis SELLAMI \\ Research Unit C3S \\ School of Sciences and Technologies, ESSTT \\ Tunis, Tunisia \\ anis.sellami@esstt.rnu.tn
}

\begin{abstract}
In Arid or semi-arid regions the ensoleillement is important while the water quality is bad. The coupling between Reverse Osmosis Desalination (ROD) processes and photovoltaic energy sources is a best solution to produce drinkable water from brackish water. However, the high intermittence of the photovoltaic energy level is the mean reason of membranes filling; therefore it makes these plants not optimally designed and operated because only a short number of constant operation points is considered and not a changeable control strategy. The present paper deals with a novel suitable control strategy approach based on the Variable Structure Model Reference Adaptive Control (VSMRAC) applied to ROD systems fed by photovoltaic sources. The control algorithm ensures minimal value of the following error between the plant and the reference model even in the presence of intermittent sources. The performances of the proposed control are shown by simulations and validated by experimental results.
\end{abstract}

Keywords-VSMRA Control; Desalination system,s Reverse osmosis, Photovoltaic systems control

\section{Introduction}

Process control is an important part of the desalination industry that needs to be operated at the optimum conditions. The desalination is a highly complex process, so that the majority of dynamic models are of the form of multi-inputsmulti-outputs (MIMO) systems. Driving these processes is a complex problem because of the interactions between the input parameters [1].

In process control industry, most of control closed loops become insensitive to a certain parameter variations and disturbances; this is mainly attributed to the influence of one input parameter on more than an output variable which can be easily understood and implemented in practice.

In the case of unstable plants where parameters change their values with the time in a known range, the design of control laws is a complex problem and need the introduction of the combination between adaptive control and the sliding mode control although the inconvenient of the last with the Chattering phenomenon and the many works to eliminate this problem [2].

Consequently and with these two points in mind we have introduce a Variable Structure Adaptive control strategy to drive a MIMO RO desalination system described by a linear state space model. Many formulas have been derived to tune the VSMRA controllers in the literature.

The Variable Structure Adaptive Control (VSAC) for monovariable systems was employed by Furuta et al. [3] where the control laws include a feedback linear term and a switching term with equivalent control regions. Then, these works were developed by the introduction of the VSAC using a Reference Model for Single In, Single Out (SISO) systems [4]. This control strategy was extended for Single In, Multi Out (SIMO) systems by Imai [5] then generalized for MIMO systems by Chen [6], Cunha, J.P.V.S., Hsu, L., Costa, R.R. and F.Lizaralde [7] and Ardeshir [8].

Y.J. Huang, T.C. Kuo, and S.H. Chang [9]. have applied the adaptive sliding mode control for non linear systems with uncertain parameters.

New methodologies were proposed by Plestan F. Y. Shtessel, V. Brégeault and A. Poznyak,[10]. The main idea is to propose a gain commutation based solution where the adaptation algorithm is applied on the feedback term, than the control law will be

$$
U(t)=-K(t) \operatorname{sign}(\sigma(x, t))
$$

Where $K(t)$ is the adaptation law and $\sigma(x, t)$ is the sliding variable. The sliding mode is established in the system after a finite time $\mathrm{t}_{\mathrm{f}}$ when $\sigma(x, t)=0$ for $\mathrm{t} \geq \mathrm{t}_{\mathrm{f}}$

The use of this control algorithm in the case of MIMO systems presents some difficulties because of the interconnection between the gains $\mathrm{k}_{\mathrm{i}}$ of the gain vector $\mathrm{K}$. The increase of the gains values can provoke the risk of saturation.

Therefore, it is highly desirable to apply a control strategy which keeps the stability of the system.

In this paper we will firstly describe the design procedures and the theoretical elements of the control action based on VSMRA Control algorithms. In the second section, we'll present the application of this control strategy to the state space model of the ROD process. The section 3 is devoted to show the experimental results, and the performances of the following algorithms, even if the reference trajectory varies quickly. 


\section{THE VSMRA CONTROL DESIGN}

\section{A. Problem Statement}

Consider a Multi Input Multi Output (MIMO) plant (S) represented in the state space by the following mathematical model

$$
\left\{\begin{array}{l}
\dot{X}=A X+B U \\
Y=C X+D U
\end{array}\right.
$$

Where the matrix $\mathrm{A}$ is unknown and of full rank, whiles the matrix $\mathrm{B}$ is assumed to be known and partitioned as:

$B=\left[\begin{array}{llll}b_{1} & b_{2} & \ldots & b_{m}\end{array}\right], \mathrm{U}$ is an m-dimensional control vector, and $(\mathrm{A}, \mathrm{B})$ is controllable.

The reference model $(\mathrm{M})$ is represented in the state space by the linear time invariant differential equation

$$
\left\{\begin{array}{c}
\dot{X}_{r}=A_{r} X_{r}+B_{r} V \\
Y_{r}=C X_{r}+D_{r} V
\end{array}\right.
$$

Where $A_{r}$ is an asymptotically stable matrix, $B_{r}$ is a known matrix, and $\mathrm{V}$ is an m-dimensional input vector with bounded elements. The purpose is to form the control $U$ that prescribes in advance the transient process of the error between the reference model and the plant outputs.

$$
e=X-X r
$$

Where e, $X_{\mathrm{r}}$ and $\mathrm{X}$ are respectively the error vector, the reference state space vector and the process state space vector defined in the case of MIMO systems such as:

$$
\begin{aligned}
& e=\left[\begin{array}{llll}
e_{1} & e_{2} & . & e_{n}
\end{array}\right]^{T} \\
& X_{r}=\left[\begin{array}{llll}
x_{r 1} & x_{r 2} & \cdots & x_{m}
\end{array}\right]^{T} \\
& X=\left[\begin{array}{lllll}
x_{1} & x_{2} & . & x_{n}
\end{array}\right]^{T}
\end{aligned}
$$

Than the control vector $U=\left[\begin{array}{lllll}u_{1} & u_{2} & \text {. . } & u_{m}\end{array}\right]^{T}$ will force the error elements to tend asymptotically to zero at a finite time.

\section{B. VSMRA Control Alghorithm}

In order to simplify the application of the VSMRAC algorithm on a real system, we propose that the matrix $B_{r}$ of the reference model can be chosen as

$$
B_{r}=B
$$

It is further assumed that an unknown matrix $\Omega^{*}$ exists such that

$$
A+B \Omega^{*}=A_{r}
$$

The control vector components are expressed as

$$
u_{i}=-\overline{\omega_{i j}} \operatorname{sgn}\left(\sigma_{i j}\left(x_{j}\right)\right) x_{j}+v_{i}, \quad \overline{\omega_{i j}}>\left|\omega_{i j}^{*}\right|
$$

Then, the control vector $\mathrm{U}$ of the MIMO plant, is generated introducing control law

$$
U=\psi X+V
$$

Where, the feedback matrix, ${ }$ with the elements $\psi_{i j}$ are adjusted using Variable Structure (VS) approach, by designing switching function $\psi_{i j}$ as described in the following section.

Subtracting equation (3) from (2), and using equation (4), (6),(7) and (9), the model of the entire system in the error state space equation is obtained as

$$
\frac{d e}{d t}=A_{r} e+B\left(\psi-\Omega^{*}\right) X
$$

Consider a Lyapunov function of the form

$$
\Gamma=e^{T} \mathrm{M} e
$$

Where $\mathrm{L}$ is a positive definite symmetric matrix which satisfies the Lyapunov equation

$$
A_{r}^{T} L+L A_{r}=-B^{T} B
$$

Where, $\mathrm{M}$ is a positive definite symmetric matrix.

Differentiating (11) with respect to time along the trajectory (10) yields

$$
\begin{aligned}
& \dot{\Gamma}=-e^{T} \mathrm{M} e+2 B^{T} L e\left(\psi-\Omega^{*}\right) X \\
& =-e^{T} \mathrm{M} e+2 \sum_{i=l}^{m}\left[b_{i}^{T} L e \sum_{j=l}^{n}\left(\psi_{i j}-\omega_{i j}^{*}\right) x_{j}\right]
\end{aligned}
$$

\section{Stability and Switching Function design}

If we introduce a switching functions $\psi_{i j}$ described as following

$$
\psi_{i j}=-\overline{\omega_{i j}} \operatorname{sgn}\left(\sigma_{i j}\left(x_{j}\right)\right), \quad \overline{\omega_{i j}}>\left|\omega_{i j}^{*}\right|
$$

With the sliding variable dynamics

$$
\sigma_{i j}\left(x_{j}\right)=b_{i}^{T} \text { Lex }_{j}
$$

So that the sliding mode is established if

$$
\sigma_{i j}\left(x_{j}\right)=0
$$

Than the switching function has the following expression

$$
\psi_{i j}=-\overline{\omega_{i j}} \operatorname{sgn}\left(b_{i}^{T} \operatorname{Lex}_{j}\right), \quad \overline{\omega_{i j}}>\left|\omega_{i j}^{*}\right|
$$

and substitute into (13), yields

$$
\begin{aligned}
& \dot{\Gamma}=-e^{T} \mathrm{Me}+2 \sum_{i=1}^{m}\left\{b_{i}^{T} \operatorname{Le} \sum_{j=l}^{n}\left[-\bar{\omega}_{i j} \operatorname{sgn}\left(b_{i}^{T} \operatorname{Lex}_{j}\right)-\omega_{i j}^{*}\right] x_{i j}\right\} \\
& =-e^{T} \mathrm{Me}-2 \sum_{i=l}^{m}\left\{\sum_{j=l}^{n}\left[\overline{\omega_{i j}}\left|b_{i}^{T} \operatorname{Lex}_{j}\right|+\omega_{i j}^{*}\left(b_{i}^{T} \operatorname{Lex}_{j}\right)\right]\right\}
\end{aligned}
$$

The terms in the summation are always positive, therefore $\Gamma<0$ and regarding (11) it can be concluded that $\|e\|$ decreases at least exponentially. 


\section{EXPERIMENTAL RESULTS}

\section{A. Design of the ROD System}

The theory of this control strategy used for multivariable systems must be applied on a real system.

In order to verify the proposed control approach performances, we have applied the VSMRAC strategy to drive a Reverse Osmosis Desalination process.

As it is shown in figure1, The ROD system is composed by three compartments: the photovoltaic generator, the electrical adaptor and the desalination unit.

The PV generator consists of an array of photovoltaic cell modules connected in series-parallel combination to provide the desired DC voltage and current.

The adaptor converts the continuous voltage delivered by the battery to an alternative voltage in order to feed the motor pump group which provides pressurised water to the membrane.

The desalination unit is composed by three RO modules, each one of them is composed by a membrane which is constituted of a thin film composite modified polyamide type able to purify feed water containing up to $3,000 \mathrm{ppm}$ of total dissolved solids. These modules have a nominal capacity of $1500 \mathrm{~L} /$ day at $800 \mathrm{kPa}$.

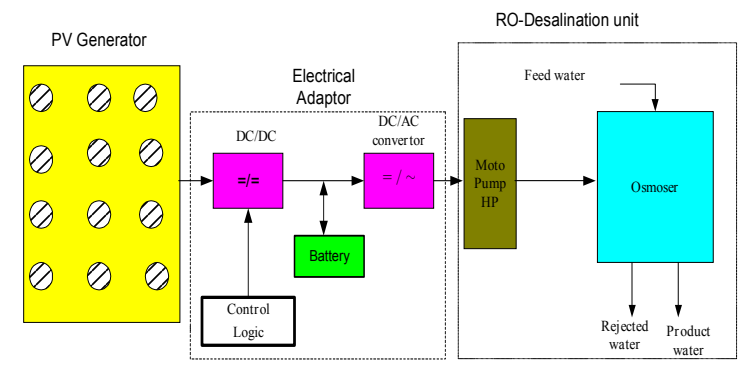

Figure 1. Design of the ROD system coupled to a photovoltaic generator

The brackish water desalination small unit is considered as a multi Input Multi Output (MIMO) system. The following configuration show the output variables or set variables which are the product flow $Q_{s}$ and the product water salinity $C s$. These two parameters are fundamentals to control water quality [11], while the input variables or manipulated variables are the motor pump angular speed $\Omega_{p}$ and the reject valve opening $\theta_{v r}$.

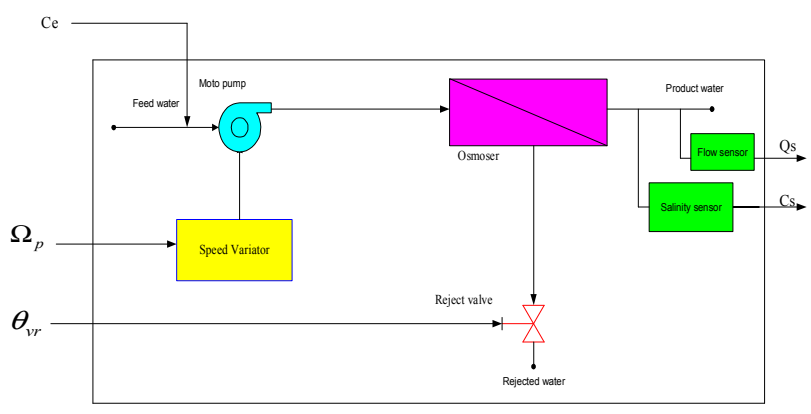

Figure 2. The desalination unit configuration

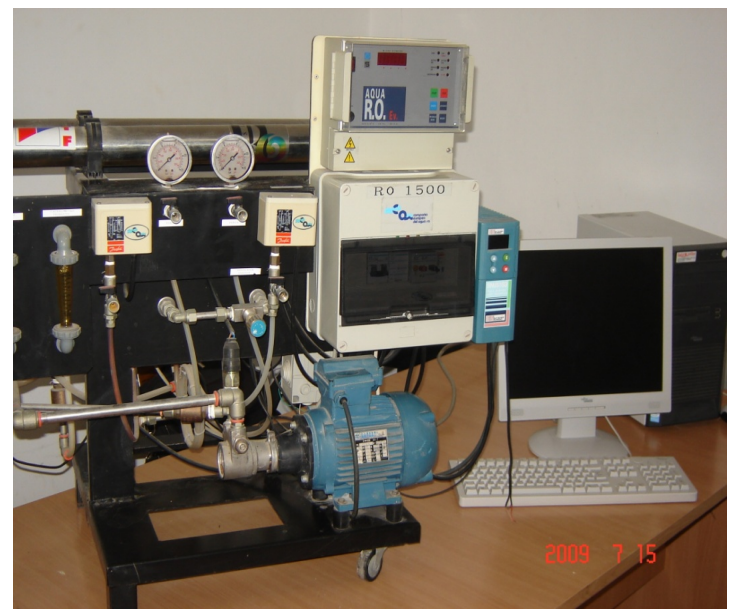

Photo 1. Photo of the experimental ROD plant located in the lab. of process control of Centre of Research and Energy Technologies, CRTEn

\section{B. The ROD System State Space Model}

The state space model of the system developed in [12] is represented by the following equation

$$
\begin{gathered}
\left\{\begin{array}{c}
\dot{X}=A X+B U \\
Y=C X
\end{array}\right. \\
X=\left(\begin{array}{l}
Q_{s} \\
\dot{Q}_{s} \\
C_{s} \\
\dot{C}_{s}
\end{array}\right) \quad U=\left[\begin{array}{c}
\Omega_{p} \\
\theta_{v r}
\end{array}\right]
\end{gathered}
$$

The constant matrices $\mathrm{A}, \mathrm{B}$ and $\mathrm{C}$ are calculated, from experimental results [.....

$$
A=\left(\begin{array}{cccc}
-1 & 1 & 0 & 0 \\
-2.25 & -1.50 & 0 & 0 \\
0 & 0 & -1 & 1 \\
0 & 0 & -4.62 & -3.23
\end{array}\right)
$$




$$
\begin{aligned}
B & =\left(\begin{array}{cc}
2.50 & 0 \\
0 & -0.56 \\
0 & -0.20 \\
-0.81 & 0
\end{array}\right) \\
C & =\left(\begin{array}{llll}
1 & 0 & 0 & 0 \\
0 & 0 & 1 & 0
\end{array}\right) \\
D & =\left[\begin{array}{ll}
0 & 0 \\
0 & 0
\end{array}\right]
\end{aligned}
$$

C. The ROD System-VSMRA Control Algorithm

The Reference model matrices are

$$
\begin{aligned}
& A_{r}=\left[\begin{array}{cccc}
-0.9 & 1.1 & 0 & 0 \\
-2 & -1.4 & 0 & 0 \\
0 & 0 & -0.9 & 1.1 \\
0 & 0 & -4.5 & -3 .
\end{array}\right] \\
& B_{r}=\left[\begin{array}{cc}
2.5 & 0 \\
0 & -0.56 \\
0 & -0.20 \\
-0.81 & 0
\end{array}\right]
\end{aligned}
$$

The calculus of the $\omega_{i j}^{*}$ values of the matrix $\Omega^{*}$ By the following expression

$$
\Omega^{*}=B^{-1}\left[A_{r}-A\right]
$$

Gives the following values:

$$
\begin{aligned}
& \omega_{11}^{*}=\omega_{12}^{*}=-0.0362 \\
& \omega_{13}^{*}=0.0141 \\
& \omega_{14}^{*}=0.0270 \\
& \omega_{21}^{*}=0.3959 \\
& \omega_{22}^{*}=0.1584 \\
& \omega_{23}^{*}=\omega_{24}^{*}=0.0566
\end{aligned}
$$

Than the matrix $\bar{\Omega}$ deduced from the matrix $\Omega^{*}$ is given by the following representation:

$$
\bar{\Omega}=\left[\begin{array}{cccc}
-0.03 & -0.03 & 0.02 & 0.03 \\
0.5 & 0.2 & 0.066 & 0.066
\end{array}\right]
$$

The VSMRAC algorithm block diagram is shown in figure 3 with the matrix $Q^{*}=\mathrm{I}$

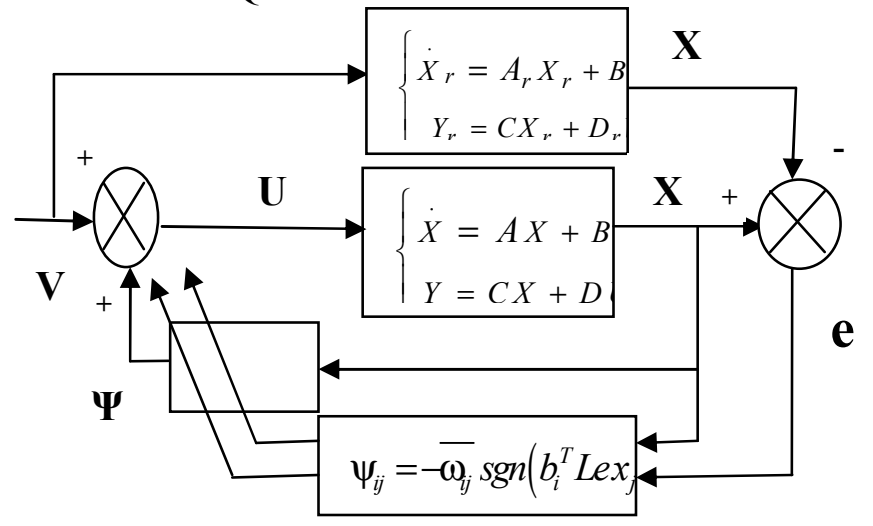

Figure 3. The VSMRA control algorithm block diagram

The results, presented in figure 4 and figure 5 show the performance of application of the VSMRA control to the desalination system, with a following error less than $2 \%$ for the water flow and less than $1 \%$ for the salinity behavior as shown in figures 6 and 7.

These results show perfect model following at a finite short time. This is the consequence of the performances combination of both of the adaptive control, real time adjusting and the robustness of the Variable Structure control.

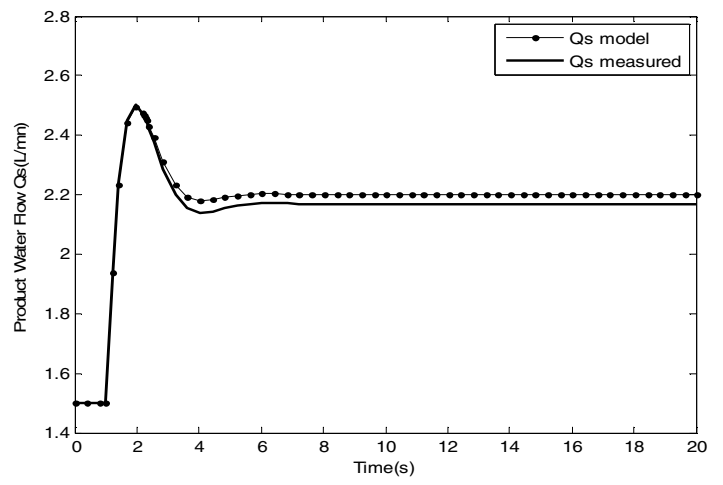

Figure 4. The product water flow rate behavior

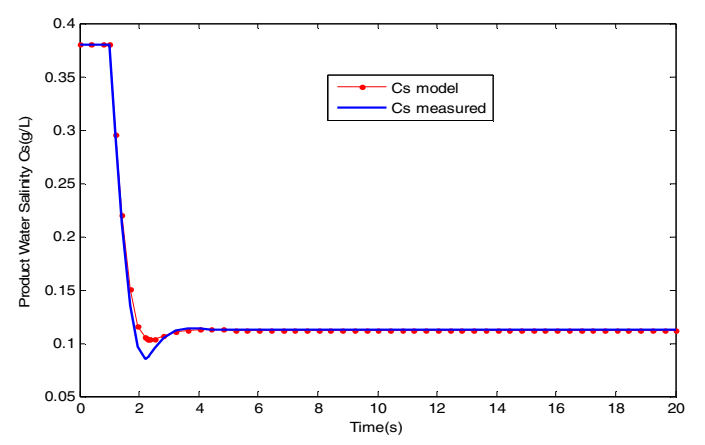

Figure 5. The product water salinity behavior 


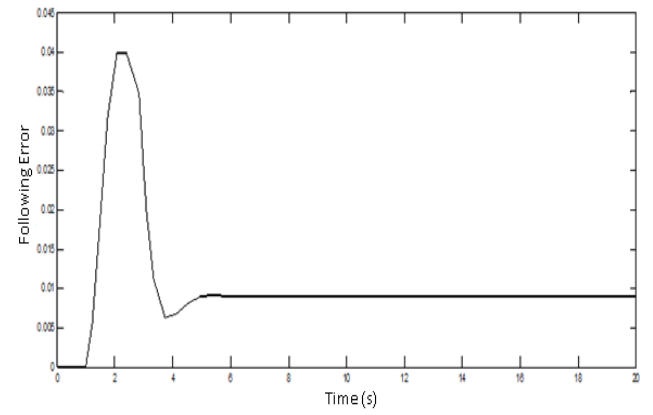

Figure6. The water flow following error behavior

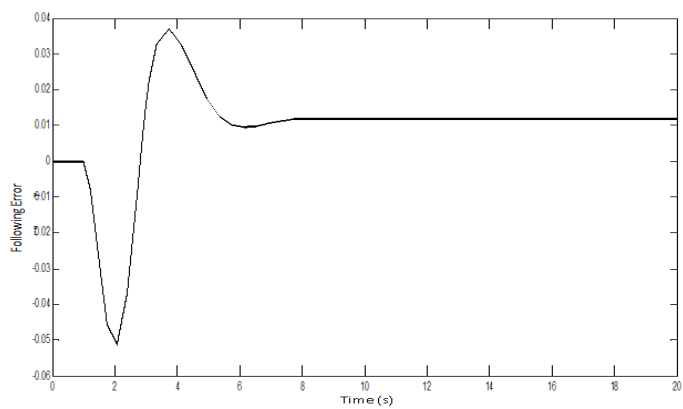

Figure7. The water Salinity following error behavior

\section{Testing of the Tracking Speed}

To study the following performances, we have chosen a fast variable model reference and show if the system can follow quickly its reference model. Figures 8 and 9 show a perfect following even in the presence of a variable reference model with a following error value is evolving around $5 \%$ as it is shown in figure 10 .

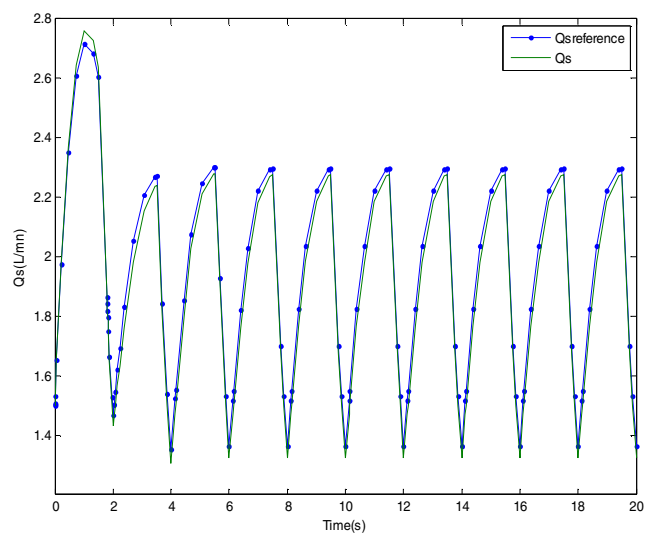

Figure 8 . The product water flow rate behavior

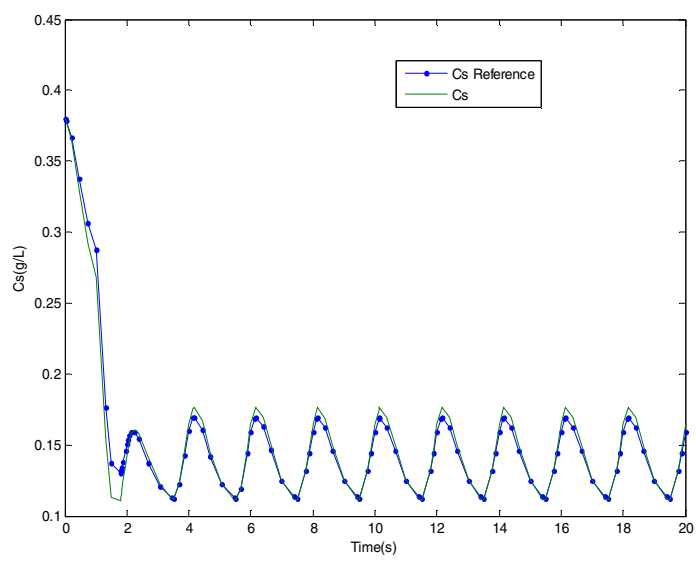

Figure 9. The product water salinity behavior

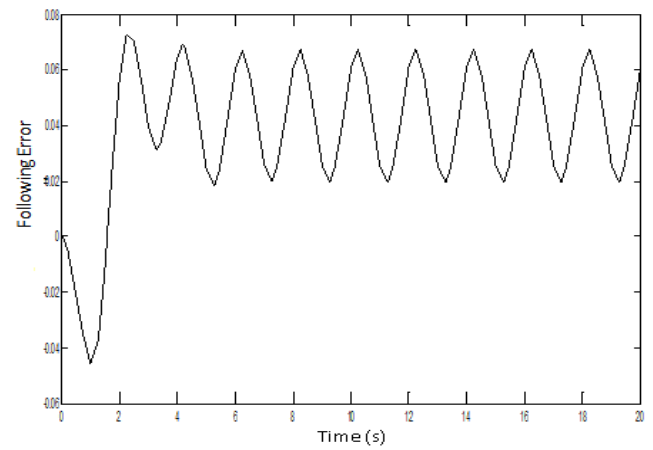

Figure10. The water flow tracking error behavior

\section{CONCLUSION}

In this paper a VSMRA Control approach has been presented. The proposed control algorithm tries to decrease the following error between the reference model and the plant outputs due to the combination of the two control types, essentially, the inherent robustness in relation to external disturbances and parameter variation. Furthermore, the VSMRAC strategy has the advantage of the flexibility to each variation of the outputs caused by the disturbed inputs such as the brackish water salinity and temperature.

The VSMRAC strategy was implemented for a RO desalination process; the results are quite encouraging and show a good set point regulation performance. However, the application of this control strategy can be extended to non linear multivariable processes.

\section{ACKNOWLEDGMENT}

This work was supported by the Center of Researches and Energy Technologies of technological pole of Borj Cedria. 


\section{REFERENCES}

[1] J. A. Mandler, "Modelling for control analysis and design in complex industrial separation and liquefaction processes," Journal of Process Control, vol.10, no. 2, pp. 167-175, 2000.

[2] H.Lee, V.I. Utkin, "Chattering suppression methods in sliding mode control systems", IEEE transaction on system, Man and Cyberneticspart B: Cybernetic, vol.38, pp.534-539, 2008.

[3] K. Furuta, "VSS type self tuning control", IEEE transaction on industrial electronics, vol. 40, pp.37-44, 1993.

[4] L. Hsu, A.D. Aranjo et R.R. Costa, "Analysis and Design of I/O based variable structure adaptive control", IEEE transaction on Automatic control, pp. 4-2, 1994.

[5] A.K. Imai, R.R. Costa, el L. Hsu, "Multivariable MRAC using Nussbaum gain" proceeding of international symposium on adaptive and intelligent systems and control, 2001.

[6] X. Chen, "Adaptive sliding mode control for discrete time multi-input, multi-output systems", Automatica, vol. 42, pp. 427-435, 2006.

[7] Cunha, J.P.V.S., Hsu, L., Costa, R.R. and F.Lizaralde," Output feedback model reference sliding mode control of uncertain multivariable systems", IEEE Transactions on automatic control,48(12), pp. 2245-2250, 2003.

[8] K. M. Ardeshir, "A variable structure MRAC for a class of MIMO systems", International J. of Mechanical Systems Science and Engineering, vol. 1 (2), pp. 4-79, 2007.

[9] Y.J. Huang, T.C. Kuo, and S.H. Chang,"Adaptive sliding mode control for non linear systems with uncertain parameters", IEEE transaction on system, Man and Cybernetics-part B: Cybernetic, vol.38, pp.534539, 2008.

[10] F. Plestan, Y. Shtessel, V. Brégeault and A. Poznyak, "New methodologies for adaptive sliding mode control", International Journal of Control, vol.83, no 9, pp. 1907-1919, 2010.

[11] M. Thomson, D. Infield, " A photovoltaic powered reverse osmosis system without battery," Desalination vol. 153, pp. 1-8, $2002 .$.

[12] A.B. Chaabene, R. Andoulsi, A. Sellami et R. Mhiri, " MIMO modeling approach for a small photovoltaic reverse osmosis desalination system " J. of Applied Fluid Mechanic, vol. 4 (1), pp.3541,2009 . 